\title{
Agôn
}

Revue des arts de la scène

7| 2015

La Distribution

\section{Emplois comiques et répertoire moliéresque : enjeux dramaturgiques d'un principe de distribution}

Céline Candiard

\section{(2) OpenEdition}

Journals

Édition électronique

URL : http://journals.openedition.org/agon/3179

DOI : 10.4000/agon.3179

ISSN : 1961-8581

Éditeur

Association Agôn

\section{Référence électronique}

Céline Candiard, «Emplois comiques et répertoire moliéresque : enjeux dramaturgiques d'un principe de distribution », Agôn [En ligne], 7 | 2015, mis en ligne le 06 novembre 2015, consulté le 04 mai 2019. URL : http://journals.openedition.org/agon/3179; DOI : 10.4000/agon.3179

Ce document a été généré automatiquement le 4 mai 2019.

Association Agôn et les auteurs des articles 


\title{
Emplois comiques et répertoire moliéresque : enjeux dramaturgiques d'un principe de distribution
}

\author{
Céline Candiard
}

1 À la lecture des dossiers pédagogiques ou des éditions scolaires des grandes comédies de Molière ${ }^{1}$, on pourrait volontiers croire que leur histoire dramaturgique n'a véritablement commencé qu'avec les mises en scène consacrées du $\mathrm{xx}^{\mathrm{e}}$ siècle : Jacques Copeau, Louis Jouvet ou Jean-Louis Barrault font alors quasiment figure de pionniers dont l'émergence miraculeuse aurait mis fin à des siècles de désert interprétatif. Lorsqu'il arrive qu'une section soit consacrée aux siècles précédents, la tendance majoritaire est de présenter l'ensemble de la période de l'Ancien Régime comme constituant un tout à peu près unifié, où les comédiens du XVIII ${ }^{\mathrm{e}}$ siècle se seraient bornés à reprendre, sous une forme quelque peu dégradée et caricaturale, les traditions de jeu qu'ils avaient reçues des premiers interprètes des rôles ${ }^{2}$. La fonction de ces évocations est alors de constituer un repoussoir, de construire historiquement un degré zéro de la complexité dramaturgique contre lequel viendraient se détacher de manière valorisante les propositions ultérieures.

Cette perception réductrice imposée par la distance temporelle et la prévalence spontanée du point de vue téléologique trouve également appui sur une conception commune de la pratique théâtrale des $\mathrm{XVII}^{\mathrm{e}}$ et $\mathrm{XVIII}^{\mathrm{e}}$ siècles comme structurée par un système d'emplois qui aurait fixé les règles de la distribution et orienté l'écriture des pièces : même s'ils ne furent formalisés que tardivement, au cours du XviII ${ }^{\mathrm{e}}$ siècle, les emplois sont généralement associés à la dramaturgie classique, dans l'idée que leur institutionnalisation n'aurait fait qu'entériner une pratique plus ancienne ${ }^{3}$. Ainsi, l'histoire des grands rôles du répertoire se diviserait en deux périodes opposées: l'époque, originelle, des emplois, qui auraient réglé la question de la distribution de manière simple et systématique en attribuant $a$ priori tous ses rôles à chaque comédien au moment de son entrée dans la troupe ; et une époque moderne aux commencements flous 
${ }^{4}$, qui correspondrait à la mise à mal du système des emplois et à son remplacement progressif par une logique de lecture dramaturgique imposée par de grandes figures individuelles d'acteurs et les premiers metteurs en scène.

3 L'inconvénient majeur de cette schématisation est qu'elle écrase la perception des mutations anciennes, fussent-elles majeures, en englobant dans une même catégorie tout ce qui est antérieur à l'entrée supposée du théâtre dans la modernité. La difficulté tient en partie à la notion même d'emploi, qui fonctionne souvent dans le discours comme une notion-écran dont on sait mal ce qu'elle recouvre précisément mais que l'on assigne de manière globale à plusieurs siècles lointains, alors qu'elle cache en réalité des conditions institutionnelles et des réalités esthétiques diverses, et qu'elle n'est apparue de surcroît que tardivement dans l'usage courant: sa première occurrence dans un dictionnaire généraliste date de la cinquième édition du Dictionnaire de l'Académie, en $1798^{5}$, et on ne la trouve employée dans des contextes professionnels que quelques décennies plus tôt. Nous tâcherons ici de comprendre les mutations qui ont conduit à son apparition et les réalités professionnelles précises auxquelles elle a pu correspondre. En prenant pour point de repère les « grandes comédies » de Molière, en particulier Tartuffe, nous verrons que c'est à la suite des transformations institutionnelles majeures liées à la création de la ComédieFrançaise, survenue après la mort du dramaturge, que se met en place une véritable logique d'emplois dans le théâtre français, et que cette nouvelle organisation du métier d'acteur influe de manière décisive sur la lecture des pièces et leur prise en charge scénique.

\section{Différencier emplois et codification des rôles}

4 Au-delà de la seule question de l'usage lexical, qui s'impose généralement a posteriori de la réalité qu'il révèle, il est malaisé de déterminer les facteurs d'apparition du système des emplois. De fait, la création de la Comédie-Française en 1680 ne les fait pas tous surgir d'un seul coup : elle ne fait qu'en créer les conditions institutionnelles. Ce n'est pas la Comédie-Française, en effet, qui impose la présence de pièces de répertoire à l'affiche des théâtres, ni la pratique d'une écriture codifiée, avec des types de rôles récurrents à l'intérieur d'un même genre dramatique. Depuis plusieurs décennies, les troupes françaises ont l'habitude de jouer tantôt des pièces nouvelles, tantôt des reprises de pièces plus anciennes qu'elles ou d'autres troupes ont déjà créées par le passé : cette alternance est une nécessité professionnelle, en particulier pour les troupes sédentaires de la capitale, qui peuvent ainsi se donner le temps d'assimiler les nouveautés sans avoir à interrompre l'enchaînement des représentations. Et la rapidité de cet enchaînement est rendue possible par la codification des genres dramatiques, qui présentent des rôlestypes récurrents aux caractéristiques spectaculaires plus ou moins stables et pris en charge de manière répétée par les mêmes acteurs au sein de chaque troupe. On observe donc déjà l'existence de spécialisations d'acteurs, le plus souvent doubles (une sérieuse et une comique), dès le début $d u \mathrm{XVII}^{\mathrm{e}}$ siècle $^{6}$ : dans toutes les troupes françaises, par exemple, au moins un comédien assume de façon récurrente les rôles d'amoureux de comédie, un autre les rôles de valets, un autre les rôles de vieillards, ou encore une comédienne les rôles d'amoureuse. C'est ainsi que la troupe de Molière a pu jouer régulièrement les tragédies et tragicomédies de Corneille, Madeleine Béjart étant depuis longtemps reconnue pour son talent tragique ${ }^{7}$, ou reprendre les comédies créées par le 
grand farceur Jodelet au Théâtre du Marais en confiant ses rôles à René Berthelot, dit Gros-René, coutumier des personnages de bouffons ${ }^{8}$.

Pour autant, ces spécialisations ne se trouvent jamais formalisées dans des documents officiels. Les quelques contrats qui nous sont parvenus n'en font le plus souvent pas mention : il y est plutôt question de la « place » de l'acteur ${ }^{9}$, terme qui renvoie à sa part de sociétaire et donc à son intéressement financier aux bénéfices de la troupe, sans précision d'un type de rôle que l'acteur devrait assumer de manière récurrente. Tout au plus se trouve parfois évoqué le privilège accordé à certains acteurs de s'attribuer prioritairement les rôles les plus en vue, comme dans l'acte de création de l'illustreThéâtre en 1643 :

Item que les pièces nouvelles de théatre qui viendront à la troupe seront disposées sans contredit par les auteurs, sans qu'aucun puisse se plaindre du rôle qui lui aura été donné; que les pièces qui seront imprimées, si l'auteur n'en dispose, seront disposées par la troupe même à la pluralité des voix, si l'on ne s'arrête à l'accord qui en est pour ce fait envers lesdits Clérin, Poquelin et Joseph Béjart, qui doivent choisir alternativement les Héros, sans préjudice de la prérogative que tous les susdits accordent à ladite Madeleine Béjart, de choisir le rôle qui lui plaira. ${ }^{10}$

6 Vedette incontestée de la troupe, Madeleine Béjart pourra se réserver le choix des premiers rôles féminins, tandis que trois comédiens (dont Molière) devront se partager les rôles principaux («Héros ») de tragédie et de tragicomédie, genres alors favorisés par la jeune troupe. Ainsi, plutôt que d'attribuer des emplois fixes, le contrat règle des questions de préséance et désigne les instances chargées de distribuer (« disposer ») les rôles à chaque nouvelle pièce, ce qui indique bien que la distribution n'est pas réglée à l'avance par des assignations permanentes et suggère même qu'elle fait l'objet de discussions, voire de contestations, parmi les comédiens. Même à la fin du siècle, lorsque le pouvoir royal fixe l'organisation de la Comédie-Italienne et de la Comédie-Française nouvellement créée par la promulgation des Règlements de la Dauphine ${ }^{11}$, il n'est jamais question que des " parts » des comédiens-sociétaires, des " places » à occuper lorsqu'elles sont laissées vacantes par le départ de membres de la troupe et des « rôles » à « disposer » au cas par cas pour chaque pièce. Le terme d' « emploi » est encore absent des usages et, si des spécialisations d'acteurs existent, elles n'ont encore aucun caractère contractuel ni astreignant.

7 En outre, à côté de cas de spécialisation caractérisée comme ceux de La Grange, qui créa la majorité des rôles d'amoureux des comédies de Molière, ou de Mademoiselle de Brie, pour laquelle le dramaturge écrivit notamment les rôles d'Agnès dans L'École des femmes, de Mariane dans Tartuffe ou encore de Dorimène dans Le Bourgeois gentilhomme, on trouve des trajectoires beaucoup plus complexes et difficilement lisibles: Molière lui-même, après s'être imposé pendant plusieurs années dans les rôles de valet fourbe ${ }^{12}$, se donna ensuite des personnages de cocus et de barbons ${ }^{13}$, mais aussi des rôles plus difficilement classables comme l'Alceste du Misanthrope ou le Chrysale des Femmes savantes. Et Louis Béjart, qui joua des rôles aussi divers que Madame Pernelle dans Tartuffe, La Flèche dans L'Avare ou encore Oronte et l'Apothicaire dans Monsieur de Pourceaugnac, semble avoir occupé dans la troupe une fonction de variable d'ajustement très éloignée d'un emploi fixe. Ainsi, s'il existe bien des spécialités d'acteurs, elles ne concernent pas tous les membres de la troupe au même degré et dépendent plutôt des capacités de jeu de chacun telles qu'elles se révèlent au cours de leur carrière que du choix définitif, à l'entrée dans la troupe, d'un personnage-type. Cela permet aux dramaturges une réelle souplesse dans l'écriture des pièces : ils écrivent alors non pas en fonction d'une liste fixe et universelle 
d'emplois qui seraient automatiquement pourvus partout, mais d'un groupe d'acteurs précis, avec ses forces et ses faiblesses. C'est ainsi, pour prendre un exemple célèbre, que Corneille écrivit le rôle de Matamore pour mettre en valeur l'acteur Bellemore, nouvellement arrivé dans la troupe du Marais ${ }^{14}$, dans ses numéros de capitan, alors même que rien dans la construction dramaturgique de L'Illusion comique (1635) ne le rendait nécessaire, et que le rôle de capitan était bien loin d'être systématiquement représenté dans toutes les troupes. Il importe donc de distinguer clairement la réalité dramaturgique que constitue la codification des rôles à l'échelle de chaque genre et la réalité professionnelle des spécialisations d'acteurs, la première n'entraînant pas nécessairement une organisation stricte de la seconde.

8 En effet, plutôt que de concevoir la codification des genres dramatiques comme une contrainte qui s'exercerait sur les comédiens, il serait plus juste d'y voir une rationalisation de la création dramatique au service des comédiens, à l'exemple de ce que les comédiens italiens avaient mis en place dans le jeu allimprovviso ${ }^{15}$ : pour faciliter le travail des interprètes, les pièces sont écrites autour de rôles-types récurrents se caractérisant non pas nécessairement par des traits psychologiques ou moraux fixes (il y a un gouffre, de ce point de vue, entre Dom Juan et le Valère de Tartuffe par exemple, alors que tous deux furent créés par La Grange) mais par leur association avec des séquences de jeu conventionnelles ${ }^{16}$. Ainsi, pour reprendre l'exemple du jeune homme amoureux, le rôle-type donne lieu de façon répétée à des scènes de confidences à son valet, de plaintes amoureuses, de déclaration, de dépit ou encore de conclusion d'un mariage; le valet, quant à lui, joue de manière récurrente des séquences de récit, d'exposition d'un stratagème, de mensonge, d'imposture ou encore de bouffonneries. Lorsqu'il écrit ses pièces, le dramaturge choisit quelques-unes des séquences-types associées au rôle pour construire tel personnage particulier de jeune homme amoureux ou de valet: de très nombreuses variations sont possibles selon le choix des séquences, l'ordre qu'il leur donne et le traitement particulier qu'il en proposera. Cela permet de comprendre qu'un même comédien puisse jouer des personnages aux caractéristiques morales très différentes sans pour autant sortir de sa spécialisation, celle-ci se définissant avant tout par des compétences de jeu : de ce point de vue, Dom Juan et Valère relèvent aussi bien l'un et l'autre du rôle de jeune homme amoureux, même s'ils ne se ressemblent nullement. Cela permet également d'expliquer qu'un même rôle-type ne soit pas traité de la même façon par les dramaturges selon la troupe pour laquelle ils écrivent : en fonction des compétences propres du comédien qui le prend en charge, un même rôle-type pourra être associé à certaines séquences de jeu plus régulièrement qu'à d'autres, et porter ainsi la marque de son interprète de manière beaucoup plus identifiable que celle de l'auteur ${ }^{17}$.

Réglant leur écriture sur les compétences des comédiens, les dramaturges disposent donc, s'agissant de la composition des personnages, d'une flexibilité égale à la leur. Il semble qu'ils furent peu nombreux à l'exploiter pleinement, la plupart des pièces donnant à voir des personnages correspondant à des rôles-types aisément identifiables. Mais la polyvalence de certains interprètes peut constituer une véritable clé de lecture pour certains personnages traditionnellement considérés comme inclassables, à l'exemple de Tartuffe. Le rôle, on le sait ${ }^{18}$, fut créé par Du Croisy, qui jouait tantôt les valets fourbes, tantôt les pédants - beaucoup plus occasionnellement les rôles secondaires d'amoureux ${ }^{19}$ ; et encore ne s'agit-il ici que de ses rôles comiques, la distribution des tragédies et tragicomédies jouées par la troupe étant très mal connue. La critique a cherché en vain à faire entrer le personnage de Tartuffe dans un rôle-type unique: malgré sa scène de 
déclaration amoureuse à Elmire (acte III, scène III), déclaration dont on a souvent souligné l'émouvante beauté ${ }^{20}$, Tartuffe n'a pas la séduction ni le succès d'un jeune homme amoureux, et Du Croisy n'en a du reste pas l'apparence physique ${ }^{21}$. Le langage dévot auquel il astreint sa parole, jusque dans ses manœuvres galantes auprès d'Elmire, pourrait s'apparenter aux divers jargons qu'assuma Du Croisy dans ses rôles de pédant, et il a comme eux pour visée d'en imposer à ses interlocuteurs. Quant à l'imposture de Tartuffe, elle n'est pas sans rappeler celles des grands valets fourbes de Molière, en particulier celle du Mascarille des Précieuses ridicules, qui englobe la quasi-totalité de ses interventions dans la pièce puisqu'il n'est comme Tartuffe démasqué qu'à la fin; mais tout « gueux $»^{22}$ qu'il a pu être, Tartuffe n'est le domestique de personne et les menaces qu'il fait peser sur la famille d'Orgon dans le dernier acte de la pièce ne s'apparentent nullement à une plaisante fourberie de valet. Si l'on se souvient que Du Croisy joua tour à tour ces différents rôles-types dans le seul répertoire moliéresque, on peut concevoir que le personnage de Tartuffe fut élaboré sur le mode de l'hybridité, en combinant des séquences de jeu de ces différents rôles comiques - auxquels il faudrait ajouter, pour la fin de l'acte IV et l'acte $V$, celui du rival de tragicomédie, auquel se rattachent les menaces et les manœuvres politiques du faux dévot $^{23}$. Un tel usage des conventions, qui deviennent ici des matériaux au service de l'inventivité dramaturgique, n'est pensable que dans un cadre professionnel souple, beaucoup moins contraignant que celui des emplois.

\section{Réorganisation professionnelle et rupture dramaturgique}

Les premières occurrences identifiées du terme d'«emploi » dans le contexte théâtral professionnel français se produisent au cours du XVIII siècle, dans des textes à valeur règlementaire. C'est ainsi qu'un ordre du 26 décembre 1743 signé du duc de Gesvres, Premier Gentilhomme de la Chambre du roi, attribue à Mademoiselle Clairon l'emploi des soubrettes en doublure de Mademoiselle Dangeville :

Nous duc de Gesvres [...] Voulant que chacun des acteurs et actrices de la ComédieFrançaise se prête à tout ce qui peut faire le bien du service, et connaissant la nécessité d'avoir plus d'une actrice pour remplir les rôles de soubrette, en expliquant en tant que de besoin, l'ordre de réception donné à la demoiselle Clairon, ordonnons qu'elle doublera la demoiselle Dangeville dans les rôles de son emploi. ${ }^{24}$

11 La même pratique s'applique aux rôles de tragédie, comme on peut le voir dans cet ordre de septembre 1779 signé par le duc de Duras, lui aussi Premier Gentilhomme de la Chambre :

Nous, maréchal duc de Duras... Ordonnons à la demoiselle Raucourt de prendre l'emploi des Reines au Théâtre de la Comédie-Française et d'y remplir en outre tous ceux où elle sera jugée nécessaire. ${ }^{25}$

12 En quelques décennies, entre la fin du XVII ${ }^{e}$ siècle et le milieu du XVIII ${ }^{e}$ siècle, une nouvelle réalité professionnelle s'est manifestement imposée qui assigne à chaque acteur un rôletype précis, de façon fixe, sous le contrôle du pouvoir royal. Le changement par rapport à la situation précédente est très net, et d'autant plus surprenant qu'il s'est produit de manière progressive, selon une logique professionnelle pragmatique plutôt que selon une volonté concertée. 
13 Sans que l'on puisse dater de manière précise l'apparition du terme et de cette pratique, il semble qu'elle fasse suite à la création en 1680 de la Comédie-Française, issue de la réunion des trois troupes permanentes qui avaient dominé la vie théâtrale parisienne au cours des décennies $1660-1670^{26}$. Cette troupe aux effectifs sans précédent n'est pas, en effet, sans poser des difficultés de distribution : si la fusion occasionne quelques départs, elle crée de nombreuses situations de doublon en réunissant plusieurs acteurs spécialisés dans le même rôle-type. Ce sont ces nouvelles questions que le Règlement de la Dauphine s'efforce de résoudre, en établissant notamment que les acteurs ayant créé un rôle précédemment le conservaient, que dans le cas des pièces nouvelles les auteurs décidaient de la distribution principale tandis que la troupe désignait les doublures, et que le Premier Gentilhomme de la Chambre du Roi serait chargé d'arbitrer les contestations en dernière instance ${ }^{27}$. Or, à en juger par les ordonnances royales successives qui furent rendues au cours des années suivantes ${ }^{28}$, ce règlement fut loin de résoudre toutes les difficultés de distribution. Ainsi se pose de plus en plus souvent le cas de rôles dont l'interprète original est mort ou a quitté la troupe. Si le Règlement de la Dauphine prévoit que la distribution soit décidée collégialement par les comédiens, il semble que les contestations aient été tellement nombreuses et inextricables que la décision fut finalement confiée aux Gentilshommes de la Chambre, c'est-à-dire au pouvoir royal $^{29}$.

14 Ainsi, pour remédier à l'absence d'une autorité légitime au sein de la troupe pour décider de l'attribution des rôles, le mode d'organisation des troupes françaises étant traditionnellement collégial $^{30}$, on a recours à l'autorité politique et à l'outil juridique : l'attribution des rôles devient alors l'objet d'une réglementation objective pour limiter autant que possible les cas de contestation. Parallèlement, on institue une pérennité : une fois un rôle attribué, le titulaire conserve pendant toute sa carrière un droit permanent sur lui, même s'il doit laisser à son « double » une part minimale des représentations. Il semble qu'au fil des années, cette attribution de rôles au cas par cas soit devenue l'attribution plus globale d'un rôle-type, attribution que l'on désigne alors par le terme d'« emploi ». On comprend aisément les avantages pratiques d'une telle généralisation : elle permet d'objectiver les principes de distribution, de leur donner une cohérence et d'éviter que chaque pièce fasse potentiellement l'objet d'une nouvelle contestation. Elle constitue ainsi une nette simplification du travail de la troupe et des Gentilshommes de la Chambre, auxquels revient également la désignation des nouveaux titulaires des emplois lorsqu'un comédien meurt ou prend sa retraite, comme l'illustrent les deux ordres cités plus haut. Il n'est donc guère surprenant que ce système ait perduré ensuite pendant plus de deux siècles dans la troupe nationale officielle et ait inspiré l'organisation d'autres troupes parisiennes et de province : le règlement du Nouveau Théâtre Italien, dès le XVIII siècle, en reprend le fonctionnement général ${ }^{31}$; sous l'Empire, le célèbre « Décret de Moscou » de $1812^{32}$ réaffirme les mêmes principes pour les quatre grands théâtres nationaux (Comédie-Française, Opéra, Opéra-Comique, Odéon) en remplaçant simplement les Premiers Gentilshommes de la Chambre par un Surintendant des spectacles assisté d'un Commissaire Impérial; il sera maintenu sous la Restauration, à quelques modifications près, parmi lesquelles le rétablissement des Premiers Gentilshommes de la Chambre ${ }^{33}$.

15 Ce qu'aucun des textes officiels ne précise, en revanche, c'est la liste exacte des emplois que les troupes devaient pourvoir ${ }^{34}$. Si le pouvoir politique tenait à régler le fonctionnement des emplois dans les troupes parisiennes, il semble qu'il ne se risqua 
jamais à en établir un catalogue. Les quelques listes d'emplois qui figurent dans les ouvrages de référence n'indiquent pas de source précise, ce qui semble significatif d'une élaboration disparate et a posteriori ${ }^{35}$. De fait, contrairement à ce qu'on observe pour le ballet et l'opéra, où l'appartenance à tel ou tel emploi repose sur des critères objectifs comme la morphologie physique ou la tessiture, le théâtre n'a pu déterminer de listes d'emplois que provisoires, dans le cadre très précis d'une troupe et d'une époque données : aucune n'a de valeur générale, la détermination des emplois étant évolutive et directement déterminée par le choix du répertoire et les grandes lignes de la création théâtrale du moment. Ainsi les nouveaux genres dramatiques, comme le vaudeville, le mélodrame ou le drame romantique au XIx ${ }^{\mathrm{e}}$ siècle, font apparaître de nouveaux rôlestypes et à terme de nouveaux emplois. Les troupes peinent parfois à faire entrer les pièces nouvelles dans leur classification, d'où la formule fréquemment employée, à la fin des contrats signés par les comédiens aux $\mathrm{XIX}^{\mathrm{e}}$ et début $\mathrm{du} \mathrm{xx}^{\mathrm{e}}$ siècles: "et tous rôles convenables à son physique et à son talent $»^{36}$. La formule est claire : l'emploi d'un acteur est une réalité fluctuante, qui dépend non pas tant de qualités physiques calibrées que de compétences professionnelles propres à chacun et susceptibles de s'adapter aux mutations de l'écriture dramatique. Corolaire logique de cette relative indétermination: l'attribution d'un emploi n'est pas pensée comme définitive et les règlements successifs prévoient les modalités des transitions, qui semble-t-il n'étaient pas exceptionnelles ${ }^{37}$. Il s'agit donc moins d'une catégorie esthétique ou dramaturgique que d'une structuration pratique du métier de comédien, rendue nécessaire par les effectifs élevés des troupes et les rivalités nombreuses qui en découlaient.

Pour autant, il serait erroné de séparer absolument la notion d'emploi des domaines esthétique et dramaturgique : en s'imposant dans la pratique théâtrale, les emplois ont de fait infléchi l'écriture des pièces et l'interprétation du répertoire de manière marquée. Dans l'écriture dramatique, l'effet le plus immédiat de la création de la Comédie-Française et de l'apparition de la logique des emplois a été d'uniformiser les rôles dramatiques, y compris et surtout ceux qui jusque-là avaient été façonnés par des interprètes individuels. C'est notamment le cas des rôles de valets, qui à l'exemple des tipi fissi italiens étaient jusque-là étroitement liés, dans leurs caractéristiques physiques, leur manière de parler et les séquences de jeu qui leur étaient consacrées, aux comédiens pour lesquels ils avaient été écrits, à l'exemple du comédien de l'Hôtel de Bourgogne Raymond Poisson pour Crispin ou de Molière pour Mascarille ou Sganarelle. À partir des années 1680-1690, les valets de comédie se multiplient et s'uniformisent sur le modèle du valet fourbe, ce qui facilite le rythme soutenu des créations et le remplacement d'un comédien par sa doublure. Cette uniformisation se traduit du reste sur le plan visuel par l'adoption d'un costume unifié, la livrée ${ }^{38}$, alors que les valets étaient jusque-là vêtus de costumes fantaisistes, plus proches des costumes de commedia dell'arte que d'un quelconque réalisme social ${ }^{39}$.

L'impact de l'apparition des emplois se fait également sentir sur la manière dont les pièces $d u$ répertoire sont lues et prises en charge par les acteurs. Une fois institutionnalisée la pratique des doublures, qui signifie que deux interprètes différents joueront le même rôle en alternance au sein d'un même spectacle et au cours de la même période ${ }^{40}$, une standardisation au moins relative du rôle devient nécessaire : le titulaire et sa doublure doivent s'accorder sur un même costume ${ }^{41}$ et des options de jeu similaires afin de ne pas perturber le spectacle d'ensemble lorsque la doublure remplace le titulaire du rôle. C'est vraisemblablement de cette époque que datent les fameuses traditions de 
jeu qui furent longtemps présentées comme remontant aux créateurs des grands rôles du répertoire ${ }^{42}$ et transmises par le biais des dynasties familiales d'acteurs ayant traversé l'Ancien Régime : avant que n'apparaisse la nécessité d'un jeu homogène, ces traditions n'avaient guère de raison d'être, les troupes parisiennes cultivant au contraire l'image d'une identité actoriale forte et spécifique à chacune ${ }^{43}$.

Si l'histoire des représentations de Tartuffe aux $\mathrm{XVIII}^{\mathrm{e}}$ et $\mathrm{XIX}^{\mathrm{e}}$ siècles donne à voir d'étonnantes variations selon les époques et les interprètes ${ }^{44}$, c'est aussi en raison de la logique des emplois, qui exige que l'on fasse correspondre un rôle à un emploi donné. Dans le cas d'un personnage hybride comme Tartuffe, cette injonction ne peut aboutir qu'à une hésitation insoluble: d'abord classé comme relevant de l'emploi des valets, qui était devenu l'emploi comique principal de Du Croisy à l'époque de la Comédie-Française, le rôle fait l'objet d'une interprétation farcesque et grimacière au cours du xviII siècle (avec Armand, Augé, Deschamps), avant d'être rendu plus sérieux et raffiné après la Révolution par des acteurs plus spécialisés dans les rôles de jeunes premiers et de petitsmaîtres (Molé, Fleury, Baptiste Aîné, Michelot), puis de passer à l'époque romantique du côté du drame, voire du mélodrame, en devenant un personnage cynique, grave et inquiétant (Damas, Geffroy, Ligier, Beauvallet). La structuration des troupes en emplois semble donc avoir été, dramaturgiquement parlant, appauvrissante, conduisant les acteurs à pousser l'interprétation du personnage dans une seule direction marquée, si bien que l'histoire du rôle se résume à une succession de propositions univoques, forcées et mutuellement incompatibles.

19 L'avènement de la mise en scène a certes permis d'introduire des nuances et de l'ambiguïté là où le système des emplois privilégiait le choix d'une seule option. Cependant, le long règne des emplois a certainement contribué à créer des réflexes durables qui leur ont largement survécu, comme celui qui consiste à chercher pour les grands rôles une clé de lecture unique, qu'elle relève ou non d'un emploi ${ }^{45}$ : la pensée dramaturgique française demeure ainsi tributaire d'un système dont elle considère généralement s'être affranchie depuis longtemps. On le voit même ressurgir dans des formes de distribution en apparence aussi éloignées que possible de la logique des emplois, comme dans la mise en scène de Tartuffe par Gwenaël Morin, au Théâtre du Point du Jour à Lyon (2013), où les stéréotypes de jeu associés aux emplois traditionnels fournissaient aux comédiens des appuis commodes pour aborder des rôles qui leur étaient échus par tirage au sort, et donnaient également aux spectateurs le moyen de les identifier aisément: il en résultait un spectacle clair et dynamique, prodigieusement lisible, mais au prix d'un renoncement assumé à une véritable complexité dramaturgique des personnages. Sans doute en raison d'une assimilation abusive entre conventions dramatiques et emplois, la mise en scène contemporaine des grandes pièces du répertoire ne semble plus percevoir, entre partis-pris univoques et désintégration du sens, l'existence ancienne d'une troisième voie, appuyée sur la souplesse du code dramatique et la polyvalence des comédiens : celle de l'hybridation, qui fait des conventions un usage à la fois intelligible et inventif pour faire surgir, au gré des intentions du dramaturge, des significations renouvelables à volonté. 


\section{NOTES}

1. Pour ne donner que quelques exemples, voir Jean-Benoît Hutier, Tartuffe de Molière, Paris, Hatier, coll. Profil d'une œuvre, 1993, pp. 75-77; dossier pédagogique de la mise en scène de Laurent Vercelletto en 2012 (http://www.abcdijon.org/12-13/ESPACEPEDAGOGIQUE/ dossierspedagogiques/Tartuffe2012.pdf, lien consulté le 12/05/15); dossier pédagogique de la mise en scène de Monique Hervouët en 2011 (http://banquetdavril.fr/files/dossierpedagogiquemaj.pdf, lien consulté le 12/05/15).

2. Voir par exemple le dossier pédagogique de la mise en scène de Tartuffe par Stéphane Braunschweig en 2008 à l'odéon, p. 33 ( http://www.theatre-odeon.eu/fichiers/t_downloads/ file_335_dpd_Tartuffe.pdf ) : « Au XVIII ${ }^{\mathrm{e}}$ s., l'interprétation n'avait fait qu'accentuer jusqu'aux dernières extrémités de la grossièreté le parti pris farcesque qu'avait pris initialement L'Illustre Théâtre... » Voir aussi, dans l'édition scolaire de la pièce dirigée par Françoise Ruller-Theuret (Paris, Larousse, coll. Petits classiques, 2006), les pp. 185-186.

3. Voir Michel Corvin, Dictionnaire encyclopédique du théâtre, Paris, Bordas, 1991, article «distribution »: «La distribution d'une pièce obéit souvent à des traditions qui classent les acteurs en emplois, déterminés d'après les critères de la tragédie et de la comédie classiques ».

4. Le statut du XIX ${ }^{\mathrm{e}}$ siècle, en particulier, est assez incertain : pour Patrice Pavis, il est le siècle par excellence de la domination des emplois ( la création théâtrale n’est plus obsédée, comme au XIX e siècle par exemple, par les emplois aux ordres desquels les comédiens auraient à se mettre », Dictionnaire du théâtre, Paris, Dunod, 1996, article « distribution »), tandis que pour Michel Corvin leur fonctionnement s'y trouve fortement perturbé par l'apparition de nouveaux genres dramatiques comme le drame romantique, le mélodrame ou le vaudeville (Dictionnaire encyclopédique du théâtre, Paris, Bordas, 1991, article « emploi »).

5. " On dit au Théâtre, qu'Un Acteur a l'emploi des Rois, des Valets, etc. pour dire, qu'Il joue les rôles de Rois, de Valets, etc. " (Dictionnaire de l'Académie française, $5^{\mathrm{e}}$ édition, Paris, J.J. Smith, 1798, article « emploi »).

6. Voir Julia Gros de Gasquet, « Le jeu du comédien », in Pierre Pasquier et Anne Surgers (dir.), La Représentation théâtrale en France au XVII siècle, Paris, Armand Colin, 2011, p. 184.

7. Voir en particulier Tallemant des Réaux, Historiettes, chapitre «Mondory ou l'histoire des principaux comédiens français », Pléiade, vol. II, p. 773 : «Il faut finir par la Béjard. Je ne l'ai jamais vue jouer ; mais on dit que c'est la meilleure actrice de toutes. Elle est dans une troupe de campagne ; elle a joué à Paris, mais ç'a été dans une troisième troupe qui n'y fut que quelque temps. Son chef-d'œuvre, c'était le personnage d'Epicharis, à qui Néron venait de faire donner la question. » La pièce dont il est question ici est sans doute La Mort de Sénèque de Tristan l'Hermite, créée en 1644 par l'Illustre Théâtre.

8. Voir à ce sujet le Registre de La Grange, qui consigne les représentations données par la troupe de Molière, puis la Comédie-Française de 1659 à 1685.

9. Voir par exemple l'arrêt cité par Émile Campardon, Les Comédiens du roi de la troupe française pendant les deux derniers siècles, Paris, Champion, 1879, p. 226 : «Le roi s'étant fait représenter le contrat passé le 30 avril 1660 entre Nicole Gassot [...] et Raymond Poisson, par lequel contrat ladite Gassot, autorisée de son mari, a quitté la place qu'elle occupait dans ladite troupe à Victoire Guérin, femme dudit Poisson, pour en jouir aux gages, appointements, droits et profits accoutumés, moyennant la somme de mille livres de pension viagère... »

10. Contrat de création de la troupe de l'Illustre-Théâtre, passé devant notaire le 30 juin 1643, et dont le texte est reproduit dans l'ouvrage de Madeleine Jurgens et Elizabeth Maxfield-Miller, Cent 
ans de recherches sur Molière, sur sa famille et sur les comédiens de sa troupe, Paris, Imprimerie Nationale, 1963, p. 224-226.

11. Celui de la Comédie-Italienne est promulgué en 1684 (on le trouve reproduit dans l'ouvrage d'Émile Campardon, Les Comédiens du roi de la troupe italienne pendant les deux derniers siècles, vol. 2, Paris, Berger-Levrault, 1880, pp. 225-230), celui de la Comédie-Française en 1685 (on en trouve le texte dans l'ouvrage des frères Parfaict, Histoire du théâtre français depuis son origine jusqu'à présent, vol. 12, Paris, Le Mercier, 1747, pp. 465-471).

12. Sganarelle dans Le Médecin volant, Mascarille dans L'Étourdi (1655), Le Dépit amoureux (1656) et Les Précieuses ridicules (1659).

13. Sganarelle dans Sganarelle (1660), L'École des maris (1661) et Le Mariage forcé (1664), Arnolphe dans L'École des femmes (1663), Orgon dans Tartuffe (1669), pour n'en citer que quelques-uns.

14. Voir Tallemant des Réaux, Historiettes, chapitre «Mondory ou l'histoire des principaux comédiens français ", Pléiade, vol. II, p. 776 : «C'est lui qui fit venir Bellemore, dit le Capitan Matamore, bon acteur. "

15. Voir à ce sujet Claude Bourqui, La Commedia dell'arte, Paris, Armand Colin, 2011, pp. 15-20.

16. Marion Faure-Ribreau observe le même phénomène dans la dramaturgie comique romaine, construite autour de « modules » récurrents (voir notamment Pour la beauté du jeu. La construction des personnages dans la comédie romaine, Paris, Les Belles-Lettres, 2012, pp. 115-116).

17. Comparer ainsi Jodelet astrologue (1646) d'Antoine Le Métel d'Ouville, écrit pour le farceur Jodelet au Marais, et Le Feint astrologue (1650) de Thomas Corneille, écrit pour Villiers : quoique réécritures de la même pièce espagnole, la première donne au valet beaucoup plus de séquences de bouffonneries. La comparaison entre Le Gardien de soi-même (1654) de Scarron, écrite pour Villiers, et Le Geôlier de soi-même (1655) de Thomas Corneille, écrite pour Jodelet, aboutit exactement au même résultat.

18. Le gazetier Robinet, dans sa Lettre en vers à Madame du 23 février 1669, donne la distribution complète de la pièce dans sa version finale de la même année.

19. Après avoir été le très épisodique Du Croisy des Précieuses ridicules, il fut par exemple le Sbrigani de Monsieur de Pourceaugnac, le Lysidas de La Critique de L'École des femmes, le Vadius des Femmes savantes et à la fois Covielle et le Maître de Philosophie dans Le Bourgeois gentilhomme (voir R.W. Herzel, The Original Casting of Molière's Plays, Ann Arbor, UMI Research Press, 1981).

20. Voir en particulier Louis Jouvet, dans Molière et la comédie classique, Paris, Gallimard, 1965, p. 250 : «Il n'y a aucune déclaration d'amour, dans aucun théâtre, qui soit aussi suave, aussi charmante que celle de Tartuffe à Elmire. »

21. On citera à ce propos le vers célèbre de Dorine ( Gros et gras, le teint frais et la bouche vermeille ", acte I scène IV), qui semble avoir été conforme à l'empâtement de cet acteur de trente-huit ans.

22. Dorine dit de lui au début de la pièce : « un Gueux qui, quand il vint, n'avait pas de souliers / Et dont l'habit entier valait bien six deniers » (acte I, scène I).

23. Sur la convention du rival de tragi-comédie, voir Hélène Baby, La Tragi-comédie de Corneille à Quinault, Paris, Klincksieck, 2001, pp. 110-111.

24. Cité par Paul Olagnier, Le Droit des artistes, interprètes et exécutants, Paris, Librairie générale de droit et de jurisprudence, 1937, pp. 117-118.

25. Idem.

26. Après la mort de Molière, en 1673, sa troupe avait été réunie à la demande du roi à celle du Marais, et une nouvelle intervention royale ordonne en 1680 la réunion de cette troupe à celle de l'Hôtel de Bourgogne pour donner naissance à la Comédie-Française.

27. Il s'agit des articles V à VIII, que l'on trouvera dans l'Histoire du théâtre français depuis son origine jusqu'à présent des frères Parfaict, vol. 12, Paris, Le Mercier, 1747, pp. 468-469.

28. Voir à ce sujet Paul Olagnier, op. cit., pp. 103-105. 
29. C'était déjà le duc d'Aumont, dans une ordonnance du 12 juin 1682, qui avait distribué les rôles créés par Molière entre les comédiens Raisin, Rosimont et Brécourt (le document, qui figure dans les archives de la Comédie-Française, est cité par Jules Bonnassies dans son ouvrage La Comédie-Française, histoire administrative (1658-1757), Paris, Didier, 1874, pp. 60-61).

30. Samuel Chappuzeau parle de "gouvernement républicain » (Le Théâtre français, Lyon, Michel Meyer, 1674, livre III, titre du chapitre XVII).

31. Voir Paul Olagnier, op. cit. pp. 120 sqq.

32. Le texte de ce décret est cité dans Recueil de décrets, ordonnances, traités de paix, manifestes, proclamations, discours, etc., de Napoléon Bonaparte et des membres du gouvernement français depuis le 18 brumaire an 8 (novembre 1799) jusqu'à la dissolution dudit gouvernement, éd. Lewis Goldsmith, Londres, R. Juigne, 1815, vol. 5, pp. 28-39

33. Voir Paul Olagner, op. cit., pp. 147-150.

34. À l'exception, logique, des textes concernant la troupe italienne, où le jeu de canevas all'improvviso est indissociable de l'assignation de rôles fixes. Contrairement à ce qui est indiqué dans l'article « emploi » du Dictionnaire du théâtre de Patrice Pavis (Paris, Dunod, 1996), le Décret de Moscou ne comporte pas de liste officielle des emplois.

35. Georges Polti, L'Art d'inventer les personnages, Paris, Montaigne, 1930, p. 49 ; Michel Corvin, Dictionnaire encyclopédique du théâtre, Paris, Bordas, 1991, article «emploi », pp. 290-291. Parmi des emplois proprement dramatiques, le premier évoque notamment l'emploi des «Dugazoncorset ", qui semble avoir été un équivalent dans l'opéra-comique des soubrettes, et le second attribue à la tragédie du XVII ${ }^{\mathrm{e}}$ siècle l'origine des "rôles à baguette ", qui désignent les rôles de magiciennes et d'enchanteresses à l'opéra aux XVIII et XIX ${ }^{\mathrm{x}}$ siècles.

36. Voir Michel Corvin, op. cit., p. 291.

37. Voir Paul Olagnier, op. cit., pp. 160-161.

38. $\mathrm{Au} \mathrm{XVIII}^{\mathrm{e}}$ siècle, on parle même de "rôle à livrée " pour désigner le rôle du valet comique : voir à ce sujet le Mercure de France de septembre 1774, p. 64.

39. Sur l'uniformisation des valets après la création de la Comédie-Française, voir Céline Candiard, Les Maîtres du jeu : le seruus ludificator dans la comédie romaine antique et le valet vedette dans la comédie en France aux XVII et XVIII siècles, thèse de doctorat, Paris, Université Paris 3, pp. 522-528. Sur les costumes, voir pp. 674-678.

40. La doublure jouait moins souvent que le titulaire d'un emploi, mais était sollicitée régulièrement, en particulier lorsque les interprètes principaux étaient en « visite » à l'extérieur, ce qui était fréquent pendant la saison d'été.

41. Voir à ce sujet l'anecdote racontée par Anne Queguiner dans son article «Pratiques du costume de théâtre, fin XVII ${ }^{\mathrm{e}}$-XIX ${ }^{\mathrm{e}}$ s. ", in Didier Doumergue, Olivier Goetz et Anne Verdier (dir.), Art et usages du costume de scène, Paris, éd. Lampsaque, 2007, p. 205.

42. «Ces jeux de scène ne remontent pratiquement jamais à Molière. Certains même sont assez récents. L'apparition, vers 1920 , de ce nouveau personnage qu'est le metteur en scène, a mis en évidence le caractère souvent factice de ces traditions, "que se sont repassées, écrit Dullin, de génération en génération les acteurs ", jeux de scène "qui, inventés dans le feu de l'action, se sont peu à peu mécanisés et n'ont plus ni drôlerie ni signification profonde ». Mais il serait faux de croire que les protestations contre cette tyrannie des traditions ne se sont élevées qu'à notre époque: en 1856, G. Planche s'indigne d'entendre répéter depuis vingt ans que seuls les Comédiens-Français possédaient la recette infaillible et ne se trompaient pas sur le sens des rôles du répertoire. » (Maurice Descotes, Les Grands rôles du théâtre de Molière, Paris, PUF, 1960, p. 9.

43. C'est ce qui ressort notamment de L'Impromptu de Versailles de Molière ou de L'Impromptu de l'Hôtel de Condé de Montfleury.

44. Voir à ce sujet Maurice Descotes, op. cit., pp. 153-167.

45. Ce réflexe concerne aussi bien les metteurs en scène (Louis Jouvet avec son Tartuffe dévot, Planchon sur l'attachement homosexuel d'Orgon envers Tartuffe, Vitez dans son parallèle entre 
Tartuffe et Théorème de Pasolini) que la critique (voir par exemple Maurice Descotes, op. cit., p. 157 : «bien que dans la troupe les emplois ne fussent pas répartis de façon rigoureuse, du Croisy tenait pratiquement celui des valets : Maître Jacques, Covielle, Sbrigani. Et cette référence situe bien le personnage de Tartuffe : un gueux. »).

\section{RÉSUMÉS}

Si l'on a tendance à schématiser l'histoire de l'interprétation des rôles en opposant à la dramaturgie moderne la pratique ancienne des « emplois » qui aurait dominé l'Ancien régime et le $\mathrm{XIX}^{\mathrm{e}} \mathrm{s}$., il faut rappeler que les emplois ne deviennent une réalité professionnelle qu'au cours de la première moitié du XVIII ${ }^{\mathrm{e}}$ siècle, à la suite de la création de la Comédie-Française : il n'existe avant cela qu'une codification des rôles qui structure l'écriture des pièces et facilite le travail des comédiens en leur permettant des spécialisations partielles, sans cadre juridique ni caractère contraignant. Après la fusion des troupes parisiennes, les effectifs sans précédent de la ComédieFrançaise créent des situations de doublon et de nouvelles rivalités qui nécessitent un réglage en amont de la distribution : c'est à ce besoin de formalisation que répond le système des emplois. Celui-ci a alors d'importantes conséquences dramaturgiques: si au $\mathrm{XVII}^{\mathrm{e}} \mathrm{s}$. les auteurs dramatiques s'adaptaient aux qualités individuelles des acteurs en présence, la structuration beaucoup plus rigide des troupes à partir $d u x \mathrm{XIII}^{\mathrm{e}} \mathrm{s}$. impose une certaine uniformisation des rôles et de l'interprétation. C'est ainsi, par exemple, que le rôle-titre de Tartuffe, écrit pour le comédien polyvalent Du Croisy, est interprété dans les siècles suivants dans plusieurs directions univoques successives, en fonction de l'emploi de ses interprètes.

Acting specialisations and their consequences on the interpretation and performance of Molière's repertory

The history of theatrical performance in France is often oversimplified through an opposition between the traditional specialisation of actors into emplois and modern dramaturgy. However, it is only in the first half of the Eighteenth Century, after the creation of the Comédie-Française, that the professional system of emplois came into existence: before then, roles were codified, and this at once structured the writing of plays and made the actors' work easier by allowing them to partially specialise, but these specialisations never had any actual legal or restrictive status. After the merging of Parisian theatre companies into one, the unprecedentedly large size of the Comédie-Française created such tense situations of redundancy and rivalry as to necessitate a systematic organisation of casting, which is why the emplois appeared. The emplois system had heavy consequences in the way plays were written, read and performed: whereas seventeenthcentury playwrights tended to adapt to the individual qualities of the actors they were writing for, the more rigid professional structures of theatrical companies from the eighteenth century brought about a certain standardisation of roles and acting. For example, the title role in Molière's Tartuffe, which had originally been written for the versatile acting qualities of $\mathrm{Du}$ Croisy, tended to be performed afterwards in several successive univocal ways, according to the specialisations of actors. 
INDEX

Mots-clés : acteur, codification, dramaturgie, emploi, répertoire, rôle

Keywords : actor, codification, dramaturgy, repertory, role, specialisation

\section{AUTEUR}

CÉLINE CANDIARD

Maître de conférences en Arts du spectacle à l'Université Lumière Lyon 2 